\title{
Evidencialidad y modalidad epistémica en dos variedades de toba habladas en Formosa, Argentina
}

\author{
María Belén Carpio \\ Raúl Eduardo González \\ Instituto de Investigaciones Geobistóricas \\ Consejo Nacional de Investigaciones Científicas y Técnicas \\ Universidad Nacional del Nordeste
}

\section{RESUMEN}

En este trabajo analizamos distintas estrategias morfosintácticas de codificación de evidencialidad y modalidad epistémica en toba del este y del oeste de Formosa, Argentina, desde la perspectiva tipológico-funcional (Chafe 1986; Bybee et al. 1994; Givón 2001; De Haan 2001, 2005; Aikhenvald 2004; etc.). En toba del este, se describe un evidencial de tipo reportativo a partir de una forma fosilizada del verbo 'decir', y el uso de dos adverbios de modalidad epistémica: Rego? Peko? 'parece que' e yataqta 'es cierto'. En toba del oeste, se analiza el uso de las raíces demostrativas más el prefijo ho-, pospuestas al elemento modificado (nombre, verbo, adverbio, o cláusula), como una estrategia de codificación de modalidad epistémica, específicamente de baja certeza subjetiva.

Palabras clave: fuente de información, certeza epistémica, estrategias morfosintácticas, variedades de toba de Formosa 


\section{ABSTRACT}

This paper analyzes morphosyntactic strategies that encode evidentiality and epistemic modality in eastern and western Toba (Formosa, Argentina), from the functional typological perspective (Chafe 1986; Bybee et al. 1994; Givón 2001; De Haan 2001, 2005; Aikhenvald 2004; etc.). In eastern Toba, a reportative derived from a grammaticalyzed 'say'-verb, and the use of two epistemic adverbs -?ego? Peko? 'apparently' and yataqta 'certainly'are described. In western Toba, demonstrative roots plus the prefix bo-, after the modified element (noun, verb, adverb, or clause), are analyzed as a strategy of encoding epistemic modality, specifically low subjective certainty.

Keywords: information source, epistemic certainty, morphosyntactic strategies, Toba varieties from Formosa

\section{Introducción}

En este trabajo analizamos distintas estrategias morfosintácticas de codificación de evidencialidad y modalidad epistémica en toba del este y del oeste de Formosa, Argentina. Para tal fin, describimos las principales diferencias etnohistóricas entre ambos grupos, los antecedentes sobre el tema en otras lenguas de la familia guaycurú y el corpus analizado (\$2-4). Reflexionamos acerca de las distintas perspectivas de abordaje de la evidencialidad y la modalidad epistémica, especialmente en lo que concierne a la naturaleza de su vínculo, a partir de lo planteado, desde la perspectiva tipológico-funcional, por Chafe (1986), Bybee et al. (1994), Givón (2001), De Haan (2001, 2005), Aikhenvald (2004), entre otros (\$5). Respecto del análisis de los datos en las lenguas estudiadas, en $\$ 6$, analizamos una estrategia de codificación de evidencialidad en términos de gramaticalización y la relación entre categorías tales como los demostrativos, la evidencialidad y la modalidad epistémica. Específicamente, describimos, en toba del este de Formosa, una estrategia perifrástica de evidencialidad de tipo reportativa en avanzado grado de gramatica- 
lización a partir del verbo qoyitega 'dicen'. Se demuestra el carácter gramaticalizado de este verbo al compararlo con su uso, con distinta flexión, pero también invariante, en construcciones causativas perifrásticas, y con otro verbo de decir ?eta? 'dice', que permite introducir citas directas. A su vez, se presenta el uso de los adverbios ?ego? ?eko? 'parece que' y yataqta 'es cierto'. Con respecto al toba del oeste de Formosa, presentamos, en primera instancia, los usos de los demostrativos restringidos al ámbito de la frase nominal y, luego, centramos el análisis en el uso de las raíces demostrativas más el prefijo bo-, pospuestos al elemento modificado (nombre, verbo, adverbio o cláusula), como una estrategia de codificación de modalidad epistémica, específicamente de baja certeza subjetiva. Finalmente, incluimos una breve síntesis de lo expuesto en el trabajo y perspectivas de investigación a futuro.

\section{Los tobas en la provincia de Formosa}

El toba es una lengua que pertenece a la familia guaycurú, junto al pilagá, mocoví, caduveo, y las ya extintas abipón y mbayá (Tovar y Larrucea de Tovar 1984: 43). En la región del Gran Chaco argentino es hablada, principalmente, en las provincias de Chaco, Formosa $y$, en menor proporción, en el este de Salta. Como consecuencia de procesos migratorios internos existen, en la actualidad, importantes enclaves en la ciudad de Rosario (provincia de Santa Fe) y en el Gran Buenos Aires. De acuerdo con los datos del último censo (INDEC 2012), la población total estimada a nivel nacional es de 126.967 personas que se autoadscriben como tobas.

Hasta fines del siglo XIX, los tobas estaban organizados en grupos cazadores-recolectores nómades que migraban estacionalmente a través de la región chaqueña (noreste de Argentina, centro-sur de Paraguay y sudeste de Bolivia). Con la ocupación de sus territorios por fuerzas militares argentinas entre 1884 y 1912, y la colonización subsiguiente, los tobas fueron violentamente forzados a instalarse de forma sedentaria y a trabajar en obrajes madereros, en la agricultura y la ganadería, y en ingenios azucareros. 
En la provincia de Formosa, se ubican principalmente en el sureste (departamentos ${ }^{1}$ Pilcomayo y Laishí, y Formosa capital), y también en el centro (departamentos Pilagá, Pirané y Patiño) —donde habitan mayoritariamente grupos pilagá-, y en el noroeste (departamentos Matacos y Bermejo) (Fabre 2006: 81-86; Wright 2002: 62-63) (véase Mapa 1).

Las principales diferencias etnohistóricas entre los grupos tobas de Formosa, es decir, entre los "tobas orientales" o takshik y los "tobas occidentales", “tobas del oeste” o ñachilamole'k — como los denominan los pilagá-, radican tanto en los grupos indígenas con los que entablaron relaciones de alianza y guerra como en la naturaleza del contacto con la población blanca o criolla. ${ }^{2}$ En este sentido, los tobas orientales fueron enemigos de grupos maká que habitaban la franja derecha del Río Pilcomayo en el Chaco paraguayo y también se consideraban enemigos de sus vecinos pilagás. Los tobas del oeste, por el contrario, fueron aliados de los pilagás, estableciendo matrimonios interétnicos e intercambios económicos de diverso tipo, además de establecer relaciones con otros grupos tobas hacia el norte de su territorio, en lo que actualmente es Bolivia. Por su parte, los tobas occidentales junto a los pilagás se enfrentaban a los chulupíes o nivaclés que ocupaban la franja izquierda del Pilcomayo, río arriba de sus territorios. Por otro lado, los territorios orientales de la provincia de Formosa fueron objeto de exploración e intentos de ocupación por parte de los españoles ya desde los siglos XVII y XVIII por medio de diversas estrategias como las siguientes: ocupación militar, fundación de pequeñas ciudades agrícolas y establecimiento de misiones jesuíticas. El oeste de la provincia, en cambio, permaneció durante mucho tiempo inexplorado y fuera de los circuitos económicos hasta mediados del siglo XX. A diferencia

1 Los “departamentos" constituyen una división territorial de segundo orden dentro de cada provincia que integra el país, excepto en la provincia de Buenos Aires y en la Ciudad Autónoma de Buenos Aires, donde se los denomina "partidos" y "comunas", respectivamente.

2 Nos referiremos a los tobas del este y centro-este de Formosa y su lengua como "toba del este" y a los tobas del oeste de Formosa y su lengua como "toba del oeste”. 
de los tobas del este, los tobas del oeste tuvieron que enfrentarse y competir con otros grupos étnicos dentro de su propio territorio (Mendoza y Wright 1989: 245). Las diferentes dinámicas de contacto de los pueblos tobas de Formosa con otros pueblos indígenas y con la población criolla permiten explicar la mutua (in)inteligibilidad entre variedades de toba y en relación a otras lenguas de la familia guaycurú. Es decir, las variedades lingüísticas habladas en el este y centro-este de Formosa, por un lado, y en el oeste de Formosa, por el otro, son mutuamente ininteligibles. La mutua inteligibilidad se observa entre las variedades habladas por los tobas en el este y centro-este de Formosa y en la provincia de Chaco, y entre los tobas del oeste formoseño y los pilagá.

Mapa 1. Localización geográfica de los tobas del este y del oeste de Formosa (adaptado de Gordillo 2006: 13)

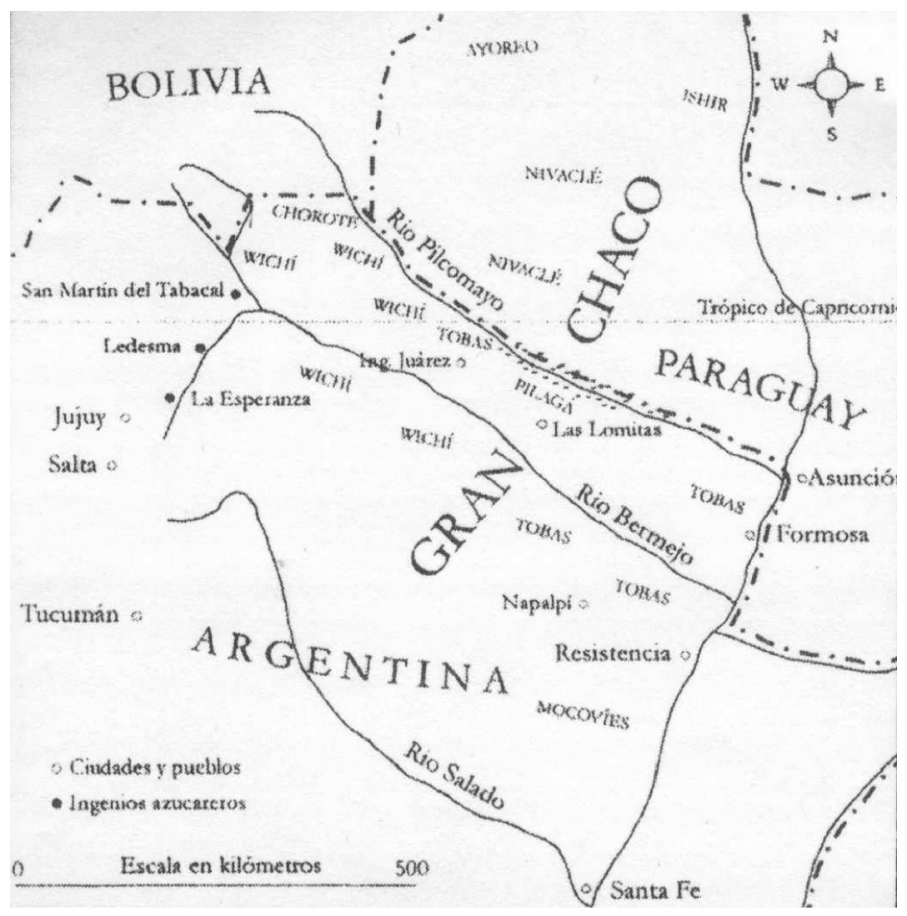




\section{Corpus}

La modalidad epistémica y la evidencialidad son analizadas a partir de textos libres y oraciones recolectados en el barrio Nam Qom, enclave periurbano situado a $11 \mathrm{~km}$ de la ciudad de Formosa, en la excolonia secular Bartolomé de las Casas (departamento Patiño) y en la comunidad de Vaca Perdida (departamento Bermejo). Los textos grabados a partir de la proyección de videos y/o imágenes han sido la principal fuente de datos para describir la codificación de baja certeza subjetiva. Dado que los participantes y/o situaciones presentados en los estímulos visuales eran desconocidos, los hablantes explicitaron frecuentemente su incertidumbre respecto de la identidad de los mismos.

\section{Antecedentes}

Las lenguas de la familia guaycurú no presentan un sistema de tiempo y modo codificado en el verbo, aunque distinguen entre aspecto progresivo y no-progresivo a través de sufijos verbales. Respecto de las descripciones previas de la modalidad y categorías afines en lenguas de esta familia, Vidal y Klein (1998) proponen una correlación entre el uso de los clasificadores ga? ka 'distal', en pilagá y toba de la provincia de Chaco, respectivamente, y la posible inferencia por parte del oyente de que el evento descrito es hipotético, no realizado o futuro. Por su parte, Messineo (2003: 167) plantea que, en toba hablado en la provincia de Chaco, todas las raíces deícticas permiten codificar, además de la configuración del referente y su relación espacial con respecto al hablante, "evidencialidad inferida”. Es decir, na 'en movimiento, próximo', so 'en movimiento, distante', $d a$ 'extendido, vertical', ñi 'no extendido, tridimensional' y $3 i$ 'extendido, horizontal' son “constatativas" puesto que implican —en determinados contextos-que el hablante es o ha sido testigo de los eventos que enuncia, mientras que $k a$ 'no perceptible, ausente' implica que el hablante no ha constatado por sí mismo los sucesos que enuncia. A su vez, Messineo (2003: 168) 
detecta el uso del demostrativo $k a$ en combinación con el verbo de 'decir' qoyenak, marcado con el morfema qo- que se designa como "persona indeterminada", para indicar que la información no ha sido constatada directamente por el hablante sino obtenida 'de oídas' (hearsay). Por ello, denomina a este tipo de estrategia reportativa. La forma verbal detectada por la autora es muy similar a la que hemos identificado en toba del este de Formosa.

Messineo (2003: 169) describe, además, el uso del sufijo -o?, fundamentalmente junto a verbos, con el fin de codificar "evidencialidad indirecta", es decir, que la información que brinda el hablante no ha sido verificada por él mismo, sino obtenida de oídas. En un estudio más reciente, el morfema -?o es analizado en el marco del proceso de gramaticalización del aplicativo - 20 ' 'debajo' (Censabella 2011: 55-60). Esto produce que, en textos libres narrativos, el aplicativo aparezca con rastros de erosión fonológica y con una nueva función: con verbos dicendi y conectores causativos se lo utiliza como un marcador catafórico de prominencia discursiva. Es decir, indica la 'importancia' o 'prominencia' de la información expresada en las cláusulas que siguen en el relato.

\section{Marco teórico}

Dentro de la perspectiva teórica tipológico-funcional, existen diferentes propuestas respecto de la relación entre la evidencialidad y la modalidad epistémica, en tanto categorías gramaticales.

Siguiendo lo propuesto por Givón (2001: 327), los sistemas de evidenciales permiten codificar la fuente de información o evidencia disponible para la aserción. La fuente de información puede ser la experiencia directa — visual, auditiva, otras - o la inferencia. A través de la modalidad se codifica, según Givón (2001: 300), la actitud o los juicios de tipo epistémico o deóntico del hablante acerca de la información proposicional expresada en la cláusula, sin incidir en la estructura argumental de la misma. Los juicios epistémicos se refieren al grado de verdad, probabilidad, certeza o evidencia que posee el hablante, mientras que los juicios deónticos se vinculan con 
la preferencia, la intención, el deseo o la manipulación por parte del hablante. Bybee et al. (1994: 178-213) plantean que las modalidades epistémicas señalan la medida en que el hablante se compromete con la verdad de la proposición y pueden codificar, en un grado creciente de certeza subjetiva, posibilidad - la proposición puede ser verdadera-, probabilidad — mayor tendencia a que la proposición sea verdadera- - certeza inferida — el hablante posee buenas razones para creer en la veracidad de la proposición-. Según Givón (2001: 326), la evidencialidad y la modalidad epistémica se superponen, aunque en muchas lenguas del mundo son codificadas por dos subsistemas distintos. En este sentido, propone la siguiente cadena de relación entre ambas categorías: Fuente de evidencia $>$ Fuerza de la evidencia>Certeza epistémica. Es decir, la información obtenida directamente por el hablante, en consecuencia, estará dotada de mayor fuerza que aquella obtenida de manera indirecta y, por lo tanto, la certeza epistémica será mayor.

Otros autores como Chafe (1986: 262) y Mushin (2001: 18) establecen dos nociones semánticas sobre la evidencialidad: (i) en sentido estrecho, como simple fuente de información expresada por el hablante para señalar o indicar el origen de la evidencia que posee al realizar su aserción, y (ii) en sentido amplio, como indicadora de la actitud epistémica que adopta el hablante frente a su enunciado, es decir, el grado de certeza subjetiva que posee en relación al mismo. En este sentido, estos autores también consideran el estrecho vínculo entre evidencialidad y modalidad epistémica, y establecen que el abordaje de ambas categorías gramaticales se encuentra necesariamente relacionado.

Por su parte, De Haan (2001, 2005) considera la evidencialidad y la modalidad epistémica como dos categorías separadas, antes que unidas a partir de un vínculo necesario. Según este autor (2001: 201-203), la modalidad epistémica representa solamente uno de los aspectos de la evidencialidad y el vínculo entre la fuente de información y el nivel de certeza del enunciado es una relación de segundo grado, solamente inferida y no necesaria. A diferencia de la modalidad, señala De Haan (2005: 379-380), la evidencialidad es 
una categoría deíctica, ya que su función básica es marcar la relación entre el hablante y la acción o evento descritos, del mismo modo que los demostrativos establecen relaciones entre el hablante y diversos objetos. La evidencialidad se divide en dos categorías principales: (i) directa, cuando el hablante ha sido testigo directo (visual, auditivo o potencialmente a partir de otros sentidos) de la acción o evento, e (ii) indirecta, cuando el hablante no fue testigo directo, sino que infiere o deduce a partir de otros (evidenciales de tipo “inferencial”), o bien lo escucha de otra fuente (evidenciales reportativos o citativos).

De Haan $(2001,2005)$ y Mushin (2001) consideran que la evidencialidad propiamente dicha se establece como categoría morfológica o bien con algún grado de gramaticalización. Al respecto, Aikhenvald propone denominar "estrategias de evidencialidad" a los elementos léxicos o perifrásticos cuya función semántica es establecer la fuente de información o fuente de evidencia de parte del hablante (2004: 10). Los evidenciales reportativos o citativos son, frecuentemente, formas gramaticalizadas del verbo 'decir' (De Haan 2001: 203, 2005: 379; Heine y Kuteva 2002: 265). Entendemos por gramaticalización el proceso que permite que determinada forma o construcción de tipo léxica asuma una función gramatical, o bien que un morfema o construcción asuma una función aún más gramatical. Se trata de un proceso irreversible, gradual y, a la vez, unidireccional. En la gramaticalización, las unidades sintácticas atraviesan, entre otros, distintos momentos: (i) pierden autonomía sintáctica, (ii) debilitan su significado referencial, (iii) suelen erosionarse o debilitarse fónicamente y perder peso fonológico, (iv) debilitan o pierden la distribución sintáctica contextual originaria, (v) suelen cambiar su estatus gramatical e integrarse a nuevos paradigmas. Al mismo tiempo, las formas ganan en cohesión gramatical con otros morfemas, adquieren significado gramatical más abstracto y la capacidad de ocurrir en nuevos contextos más generales, alejados de los etimológicos originarios. En un doble y simultáneo proceso de pérdida y ganancia, el cambio es visto como acumulativo, de manera que los nuevos valores más abstractos y más 
gramaticales pueden coexistir con las formas y significados léxicos de base (Heine y Kuteva 2002: 2; Company Company 2009: 71-72). Por ejemplo, en los datos analizados, el verbo 'decir' en su forma gramaticalizada ocurre como modificador de nombres, especificando la fuente de la información, función que no desempeña como verbo pleno.

A modo de hipótesis planteamos que las estrategias morfosintácticas de codificación de la evidencialidad y la modalidad epistémica en toba del este y del oeste de Formosa permiten vislumbrar un vínculo no necesario, sino inferido entre evidencialidad y modalidad epistémica.

\section{Análisis de los datos}

Describimos las distintas estrategias morfosintácticas, detectadas en el corpus analizado, que permiten a los hablantes de toba del este y del oeste de Formosa expresar la fuente de la información y distintos grados de certeza subjetiva.

\subsection{Toba del este de Formosa}

En toba del este, analizamos una estrategia de evidencialidad de tipo perifrástica en avanzado grado de gramaticalización a partir del verbo qoyitega 'dicen'. Demostramos el uso de verbos de 'decir' tanto en la codificación de la evidencialidad reportativa como en las citas directas. A su vez, describimos el uso de dos adverbios de modalidad epistémica, que codifican un bajo y un alto nivel de certeza sobre la veracidad de la proposición por parte del hablante: ?ego? Peko? 'parece que' e yataqta 'es cierto'.

\subsubsection{Evidencialidad indirecta reportativa}

A través del uso del verbo qoyitega 'dicen' se expresa, en toba del este, que la información ha sido brindada por una fuente de información ajena al hablante, el cual no fue testigo directo de la acción o evento que describe, es decir, se trata de un evidencial de tipo reportativo o citativo. Planteamos que esta forma presenta un nivel 
avanzado de gramaticalización porque se trata de una construcción cristalizada, invariante, que siempre es codificada a través de qoyitega 'dicen', es decir, del verbo -in 'decir' — realizado como $i$ junto a los prefijos de pasiva impersonal qo- qa- ba- —realizado como qo-—, de tercera persona tipo I $y^{-}$, y a los sufijos de aspecto progresivo -tak -teg y alativo -a (1).
(1) d-an-agan na shiyaga
3I-dar-AGT DAC $\begin{aligned} & \text { persona-O } \\ & \text { COORD }\end{aligned}$
'Plantan esos hombres, sin
qo-y-i-teg-a
PIMP-3I-decir-PROG-AL feo
dicen (es) fea'

En (2)-(4) se observa que esta estrategia de evidencialidad tiende a ser utilizada cuando las cláusulas discursivamente próximas poseen verbos morfológicamente pasivizados. Como plantea Siewierska (1984: 96-100), en la construcción pasiva impersonal no es posible distinguir entre primera, segunda y tercera persona en el verbo y, en términos semánticos, se prefiere un agente humano o animado. Estos rasgos propios de la construcción pasiva impersonal la tornan idónea para contribuir a la codificación de estrategias evidenciales del tipo 'dicen' que se caracterizan por ser expresadas en tercera persona o bien por medio de formas impersonales. Si bien, en toba del este, el sufijo alativo se comporta como un aplicativo, en las cláusulas que involucran a qoyitega no se observa el argumento promovido, sino que se trata de una forma gramaticalizada similar a they say en inglés, con valor de 'dicen'.
(2) qa-y-wek
na
salta qa-y-wege
PIMP-3I-llevar DAC
salta PIMP-3I-llevar.DIR
'Llevan a Salta, llevan
na qo-y-i-teg-a l-epentak
DAC PIMP-3I-decir-PROG-AL 3POS.INAL-trabajo
dicen, a su trabajo'


En (3) se muestra un uso enfático del verbo 'decir' con valor evidencial y se observa, además, que, como en (4), qoyitega 'dicen' no solamente puede funcionar dentro de un complemento verbal, sino como verbo nuclear de la cláusula. ${ }^{3}$

(3)

$\begin{array}{llll}q 0-y-i-t e g-a & k a & \text { shiyagawa } & d \text {-aramaqna-taGan } \\ \text { PIMP-3I-decir-PROG-AL } & \text { DNP } & \text { persona } & \text { 3I-creer-NPROG.AGT }\end{array}$

'Dicen que la persona cree,

ka shiyagawa qo-y-i-teg-a

DNP persona PIMP-3I-decir-PROG-AL

la persona, dicen'

(4) na?ana

qo- $y-i-t e g-a$

qa-y-ache-tak

grabasyon

PDAC

PIMP-3I-decir-PROG-AL

PIMP-3I-llevar-PROG

grabación

'Esto dicen (que) están llevando (la) grabación'

El uso de esta forma gramaticalizada del verbo 'decir' constituye una estrategia que, si bien no es de tipo morfológica, la consideramos como evidencial por el hecho de poseer una forma fija, gramaticalizada, con una función semántico-pragmática específica. La forma fija se comprueba por la regularidad en diversos contextos y por el hecho de que el verbo -in 'decir', sin el morfema aspectual progresivo y el alativo, permite, por ejemplo, expresar causación indirecta. En el corpus analizado, se ha detectado un tipo de causativo perifrástico que también involucra al morfema de pasiva impersonal qo-, al verbo -in 'decir' junto a un verbo antipasivizado a través del sufijo agentivo -agan -ogon, que codifica al evento causado. En los ejemplos (5)-(7) puede observarse cómo opera esta causación indirecta a través del verbo 'decir'. ${ }^{4}$

3 En la lengua los determinantes demostrativos también funcionan sincrónicamente como complementizadores verbales. En (3) qoyitega es el verbo principal ya que introduce una cláusula de complemento a través del complementizador $k a$ y cuyo núcleo es da?amaqnataGan 'cree'. En (4), si bien no se observa el uso de complementizador, la forma evidencial se antepone a qayachetak 'están llevando' con lo cual establecemos que es el verbo principal dentro de una completiva paratáctica.

4 Otras estrategias de causativización en toba del este de Formosa son descritas en González (2015: 159-165). 
Carpio y González • Evidencialidad y modalidad epistémica en dos variedades de toba 133

(5) d-ekes-ogon na qo-y-in

3I-cortar-AGT DAC PIMP-3I-decir

'Le dicen que corte'

(6) s-asot-aGan

na $\quad$ qo-y-in

1I-mover los pies-AGT DAC PIMP-3I-decir

'Me dicen que patee'

(7) d-Pon-agan

3I-cantar-AGT

'Le dicen que cante' na $\quad q o-y$-in

DAC PIMP-3I-decir

No es posible conjugar esta forma del verbo 'decir', que siempre se expresa por medio de la tercera persona (paradigma defectivo) y el prefijo de pasiva impersonal qo-, lo cual nos señala que se encuentra en un avanzado proceso de gramaticalización y los hablantes, en general, lo traducen como 'hacer' más que como 'decir', con lo cual su sentido coercitivo, para inducir una acción, también se encuentra altamente convencionalizado. Esta relación con la causación indirecta la establecemos para demostrar que qoyitega es una forma gramaticalizada invariable que solamente es utilizada con valor evidencial, pero que, sin embargo, el verbo -in 'decir' en combinación con el prefijo qo- también aparece en otros contextos vinculados directamente con las rutas de gramaticalización de dicho verbo, entre ellos, la causación indirecta perifrástica como se muestra en (5)-(7).

Cuando se trata de citas directas, es decir, cuando el hablante presenta directamente la escena que desea narrar en voz de una tercera persona, en toba del este, también se utiliza un verbo de 'decir' aunque se recurre a una base verbal distinta y conjugada en tercera persona singular $(\varnothing$-?eta?). A diferencia de la evidencialidad reportativa o citativa codificada a través de qoyitega 'dicen', en las construcciones de cita directa, el verbo Peta? 'dice' concuerda en número con el referente nominal de la meta humana y no ocurre junto al prefijo de pasiva impersonal (8)-(10). 
(8) nache se?eso doqshe-liek Ø-?eta?

COORD DDAL 1SG criollo-GENT.MASC 3I-decir

"Entonces ese criollo dice así:

nagi nache hayim Ø-wagan ne?ena ll-igewo se?eso

ADV COORD 1SG 1I-golpear DDAC 3POs.INAL-entrada DDAL

'hoy, entonces, yo golpeo la entrada de esa víbora,

PadaGanaq qami? qaw-ki?-shigem qome

víbora 2PL 2I.PL-subir.2I.PL-ARRIB ADV

ustedes suben después"”

(9) se?eso doqshe-liek Ø-Peteg-a-lo so qom-lashe?

DDAL criollo-GENT.MASC 3I-decir-AL-PLPA DAL toba-GENT.PL

"Ese criollo dijo a los tobas:

qa-way-? na?a ba-Ø-wenaGa-ñi-ta

2I.PL-quedarse-2I.PL ID PIMP-3I-hacer silencio-AB-NPROG

'Quédense acá haciendo silencio,

sa ba-yapke nache Ø-ayin na balap

NEG PIMP-estar lejos COORD 1I-disparar DAC boca

está cerca entonces yo disparo en la boca'”

(10) nache sePeso doqshe-liek qoPollaga Ø-Pena-peg-a na qom COORD DDAL criollo-GENT.MASC ADV 3I-decir-IT-AL DAC tobas

“Entonces ese criollo, en aquel tiempo, les dijo a los tobas.

Ø-?eta? $\tilde{n}$-oqone-wa?a in-puesto so-ta-ygi

3I-decir 1II-ir-EXT.AL 1POS.ALIE-puesto 1I-ir-LOC

Dijo así: 'Voy hasta mi puesto, voy y

bin-do-wek qa-nallik nache qo-wita-y na?a

1II-traer-EXT 2POS.INAL.PL-comida COORD 2I.PL-quedarse-2I.PL ID traigo su comida entonces quédense acá, 
Carpio y González • Evidencialidad y modalidad epistémica en dos variedades de toba 135

hayim s-ay-ge da klorinda Ø-irigen-?a ka wataganaq 1 SG 1I-ir-DIR 1 DPA Clorinda 1I-buscar-AL DNP policía yo voy hasta Clorinda a buscar a la Policía””

6.1.2. Adverbios de modalidad epistémica

En lo que respecta a la modalidad epistémica, uno de los mecanismos de expresión que se observan en toba del este de Formosa es el uso de adverbios que codifican modalidad 'débil' y 'fuerte', respectivamente. El adverbio modal Pego? ?eko? 'parece que', expresa modalidad 'débil' o bajo nivel de probabilidad o certeza por parte del hablante (11)-(12), mientras que el adverbio yataqta 'es cierto' codifica modalidad 'fuerte' o alto nivel de certeza sobre la veracidad de la proposición (13)-(14).

(11) Reko? n-awogon-a na waka-pi na na?aq

ME 3II-arrastrar-AL DAC vaca-COL DAC día

'Parece que arrastraba a las vacas aquel día'

(12) qayqa ka Pego? n-yek da Ø-neta-ña

EXIST.NEG DNP ME 3POS.ALIE-casa DPA 3I-estar-AB.AL

'No hay parece su casa para estar (vivir)'

En (13)-(14), cuando se utiliza el adverbio de modalidad epistémica 'fuerte' yataqta 'es cierto' o 'está siendo cierto', el hablante expresa no solamente la alta certeza y el alto nivel de verdad de la proposición, sino, además, su fuerte compromiso ante lo que relata o cuenta.
(13) d-e?en-tagan
nache yataqta
bi-koma?
3I-trabajar-NPROG.AGT COORD ME
3I-saciarse
'Trabaja entonces, es cierto, se sacia,
woro da l-madaga
EXIST DPA 3POS.INAL-satisfacción
hay esta su satisfacción' 
$\begin{array}{clll}\text { (14) yataqta } & \text { qa-n-azet-?ot } & \text { se?eso } & \text { doqshe-liek } \\ \mathrm{ME} & \text { PIMP-3II-acercarse-DEB } & \text { DDAL } & \text { criollo-GENT.MASC }\end{array}$

'Es cierto, estaban cerca debajo de ese criollo'

De acuerdo con Givón, una de las características de los adverbios de modalidad epistémica es su alcance, el cual implica a toda la cláusula, a diferencia de otro tipo de formas adverbiales más prototípicas cuya función es la de modificadores de predicaciones, verbos $\mathrm{u}$ otros adverbios (2001: 92). Los adverbios ?ego? Peko? 'parece que' e yataqta 'es cierto' expresan modalidad epistémica, en toba del este, y poseen valores opuestos en el continuum de baja y alta certeza, y compromiso del hablante con respecto a su enunciado.

\subsection{Toba del oeste de Formosa}

En toba del oeste, detectamos una estrategia de codificación de modalidad epistémica que permite expresar baja certeza por parte del hablante respecto de las características del referente de un nombre, de la situación descrita en la cláusula, o del momento en el que se lleva a cabo dicha situación. El alcance de la modulación está dado por el constituyente al que se posponen las raíces demostrativas (DEM) junto al prefijo ho- 'baja certeza subjetiva'. Describimos, en primer lugar, las raíces demostrativas en su forma escueta y formando bases demostrativas, para luego contrastar su uso junto al prefijo ho- pospuestas al elemento modificado con el fin de codificar la incertidumbre o la posibilidad de que el constituyente modificado sea verdadero por parte del hablante. A su vez, se describe el uso del coordinante disyuntivo 'wota $a$ a 'o' solo o junto a bo-DEM cuando el hablante explicita una alternativa al referente o situación respecto del cual manifiesta su incertidumbre.

\subsubsection{Raíces y bases demostrativas}

Las raíces demostrativas en su forma escueta ocurren antepuestas a los nombres, y expresan los siguientes rasgos deícticos de los referentes nominales: ñi? 'sentado', da? 'parado', di? 'acostado', ga? 'ausente', ben 'próximo al centro deíctico', bo? 'alejándose del 
Carpio y González • Evidencialidad y modalidad epistémica en dos variedades de toba 137

centro deíctico' y na? 'acercándose al centro deíctico'. Reciben flexión de número (singular/plural) y, solo en singular, concuerdan en género (masculino/femenino) con el nombre al que modifican (15).

(15) a. qa'ma? qom qo-n-bo'tek ñi? pe'gaq

entonces si PIMP-3II-esquilar DSE caballo

'Entonces, si se esquilaba el caballo...'

b. b-e'qota-Ga qom'le ba-'ñi? i'yelabogo

1I-llegar-1G enseguida FEM-DSE círculo de agua

'Llegamos enseguida al círculo de agua'

c. qaw-qoli-yi-'lo $\tilde{\mathrm{n}}<\mathrm{a}>$ ? $\quad$ ta $\mathrm{G} a^{\prime} k i-$ ?

1GRI-revolver-AD-PL DSE $<$ PL $>$ olla-PL

'Revolvimos las ollas'

A su vez, las raíces demostrativas funcionan no solo adnominalmente, sino también pronominalmente cuando forman bases demostrativas endofóricas junto al sufijo -me 'endofórico' (16).

$\begin{array}{llll}\text { a. qa'lagaba' qa?li } & w o \text { ? 'ho?-me ne'dep } \\ \text { pero } & \text { cuando } & \text { EXIST DAL-ENDOFinundación }\end{array}$

'Pero cuando vino la inundación'

b. bo?-me 't-awe 'wyaq

DAL-ENDOF 3I-entrar monte

'Aquel entró al monte'

En cambio, solo cumplen función adnominal cuando integran bases demostrativas exofóricas visibles junto al sufijo - bo 'exofórico visible’ $(17)^{5}$ y únicamente pronominal en las bases demostrativas

5 La base demostrativa exofórica visible 'benho presenta un comportamiento peculiar puesto que, además del uso adnominal que presentan las demás bases demostrativas exofóricas, puede ocurrir, por sí misma, sin un nombre modificado 
exofóricas no visibles, es decir cuando ocurren junto al sufijo - ha 'exofórico no visible' (18) (Carpio 2012: 44-55).

(17) ba'yim on'le-k na'yi b-abotay't-a, ba-'ñi?-bo be'yogoda 1sG uno.DIM-MASC ahora 1I-estar con-PL FEM-DSE-EXOFVIs criolla

'Yo estoy solo ahora con esta (visible, sentada) criolla

'ñi?-bo qobe-'le?ek

DSE-EXOFVIS criollo-GENT.MASC

y este (visible, sentado) criollo'

(18) a. 'da?-ha 'd-Ponasan-tak

DPA-EXOFNVIS 3I-cantar-PROG

'Este (parado, no visible) está cantando'

b. ha-'da?-ba 'd-Ponasan-tak

FEM-DPA-EXOFNVIS 3I-cantar-PROG

'Esta (parada, no visible) está cantando'

Las bases demostrativas exofóricas visibles y no visibles podrían ser analizadas como marcadores de evidencialidad directa: visual (17) y no visual, por ejemplo, auditiva (18), respectivamente. Sin embargo, debido a su uso restringido dentro de la frase nominal, es decir, a que su alcance respecto de la fuente de información se circunscribe a los referentes de los nominales con los que ocurren o a los que reemplazan como pronombres, consideramos pertinente incluirlos dentro de la categoría de los demostrativos.

\subsubsection{Baja certeza subjetiva en cláusulas declarativas}

En el corpus analizado, las raíces demostrativas fueron observadas junto al prefijo ho-, ambos pospuestos al elemento modificado, con el fin de expresar baja certeza por parte del hablante, funcionan como marcadores de modalidad epistémica. La posición del

permitiendo el anclaje espacial de la situación descrita en la cláusula, es decir funcionando a la manera de un adverbio de lugar. 
marcador de modalidad epistémica permite identificar su alcance, es decir, la incertidumbre recae sobre el constituyente contiguo, inmediatamente antepuesto a bo-DEM.

En (19) se ilustra el uso de la raíz demostrativa da? junto al sufijo -me 'endofórico', antepuesta al nombre ad-et'?a 'tu papá', con el fin de situar espacialmente al referente de dicho nombre, y junto al prefijo ho-, pospuesta a dicho nombre, con el fin de expresar baja certeza subjetiva de parte del hablante respecto de la identidad del referente nominal.

(19) Ø-ek 'da?-me ad-et'?a bo-'da?

3I-ir DPA-ENDOF 2POS.INAL-padre BCS-DPA

'Se va tu papá, ¿será? (no estoy seguro si es tu papá)'

En este sentido, la estrategia que permite codificar el grado más bajo de certeza por parte del hablante o la posibilidad de que una proposición o un componente de la misma sea verdadero consiste, en toba del oeste, en el uso del prefijo bo- junto a las raíces demostrativas luego del elemento modificado.

En (20) se observa cómo el hablante modula el grado de aserción en cláusulas declarativas mediante el uso de la raíz demostrativa ñi? junto al prefijo bo-, pospuestos al nombre na' GanaGayk 'sembrador' dentro de la cláusula relativa puesto que no está seguro si esa es la profesión de la persona a la que se refiere.

(20) wo? ga? n-i'ki?i 'bo?ne y-alo'Gon wo? ñi? biyaga'wa EXIST DNP 3IPOS-foto REL1 3I-mostrar EXIST DSE persona 'Hay una foto que muestra que hay una persona

'bo?ne na'Ganagayk bo-'ñ $i$ ?

REL1 sembrador BCS-DSE

que es sembradora, ¿ será? (no estoy seguro)’

Las raíces demostrativas, cuando funcionan como marcadores de modalidad epistémica, concuerdan en género y número con el 
elemento modificado. Por ejemplo, cuando la entidad respecto de la cual se expresa duda es plural se observó el uso del sufijo -wa, acentuado, junto a la raíz demostrativa ga? 'ausente' más el prefijo bo-, y cuando dicha entidad es asignada al género femenino, el prefijo bo- se realiza como ha- y ocurre junto a la raíz demostrativa pospuesto al nombre modificado (21).

\author{
(21) wo'?o-y bomaGa'ki-? 'togoy-q-a bo-ga?-'wa o \\ EXIST-PL camisa-PL vieja-MASC-PL BCS-DNP-PL O \\ 'Habrá camisas viejas, ¿será? (no puedo asegurarlo), o

$\begin{aligned} & k e^{\prime} m a \mathrm{G} a h o \\ & \text { pantalón togoy vieja }\end{aligned}$ BCS.FEM-DNP
pantalón viejo, ¿̇será? (no puedo asegurarlo)'

Las raíces demostrativas más el prefijo ho-, pospuestas al elemento modificado, no solo permiten expresar baja certeza subjetiva de parte del hablante respecto de un referente nominal sino que su alcance puede extenderse a toda la proposición o restringirse a la situación descrita por el predicado verbal. En (22a) la baja certeza de parte del hablante recae sobre toda la proposición, mientras que en (22b) solo sobre el referente del nombre nogoto' lek 'niño'.
a. 'bo?ote
'n-owi? bo-' $\tilde{i} i$ ?
ya
3II-venir BCS-DSE
'Ya vino ¿será? (no es posible asegurarlo)'
b. qa'ma?le 'n-owi? 'bo?-me 'qogot bo-'bo? entonces 3II-llegar DAL-ENDOF hijo BCS-DAL
'Entonces llegó el hijo (no estoy seguro si era el hijo)'

La raíz demostrativa ñi? 'sentado’ más el prefijo bo- 'baja certeza subjetiva' ocurre, en (23), luego del verbo 'bachi 'llevo', pero antes de la frase nominal que expresa al argumento más parecido al paciente $(\mathrm{P})$. En este ejemplo, el alcance de la incertidumbre se restringe al evento expresado por el verbo. 
Carpio y González • Evidencialidad y modalidad epistémica en dos variedades de toba 141
(23) 'b-achi bo-'ñi? $\tilde{n} i$ ?
$y$-OGO'ki
1I-llevar BCS-DSE DSE
1POS.INAL-bolso

'No estoy seguro si voy a llevar mi bolso'

En (24) bo'ga? expresa baja certeza subjetiva respecto del momento en que tendrá lugar la situación descrita por el verbo y se ubica contiguo a qom'le yam' ta?ale no' lo? 'antes del mediodía'.
(24) 'qa?di h-o'widacabema qom'le yam'ta?ale no'lo? ho-'ga? así 1I-llegar.1G enseguida antes día BCS-DNP
'Así vamos a llegar antes del mediodía, ¿`erá?'

Cuando el hablante no está seguro acerca de si la mujer que acompaña al hombre de la imagen proyectada es su esposa o no, recurre al uso del prefijo ha- (forma de ho- concordante con el nombre femenino modificado) junto a las raíces demostrativas $d a$ ? 'parado' y na? 'acercándose' luego de los nombres ya' wo 'mujer' y lo'wa 'su esposa', respectivamente (25).

(25) wo? da? biyaga'wa te?n ya'wo ba-'da? wota'Ga EXIST DPA persona también mujer BCS.FEM-DPA O

'Había un hombre también una mujer, ¿será? o

$$
\text { l-o'wa ba-'na? }
$$

3POS.INAL-esposa BCS.FEM-DAC

su esposa, ¿será?’

A su vez, con muy baja frecuencia, se detectó el uso del morfema ho, sin raíz demostrativa, pospuesto al constituyente modificado, con el fin de expresar posibilidad, como se muestra en (26).

(26) wo? ga? menaGa'ki bo, qa'lasaba ha? b-ateto'n-aq
EXIST DNP negocio BCS pero NEG 1I-conocer-1G

'Había negocio, tal vez, pero no lo conocíamos 
qa? ba? b-awa'n<aG $>a \quad$ layka'wa

cuando NEG 1I-encontrar/tener/ver $<1 \mathrm{G}>$ dinero, moneda

cuando no teníamos dinero’

En construcciones en las que ocurre el verbo 'betpiga 'creo, pienso', como se ilustra en (27)-(28), las raíces demostrativas junto al prefijo bo- refuerzan la baja certeza subjetiva ya expresada por el lexema verbal.

(27) balqo'te da? y-a'chogodek ha'yim neboqo'lak

abundante DPA 1POS.INAL-sufrimiento $1 \mathrm{SG}$ jovencito

'Tenía mucho sufrimiento cuando yo era jovencito,

'b-etpiga katorse años bo-'na?
1I-creer catorce años
creo catorce años ¿será?'

(28) qa'ma? 'b-etpiga yi'ma? y-a'wik ga? 'wyaq, entonces 1I-creer todo 3CE-quemarse DNP monte 'Entonces creo que todo el monte se está quemando, 'wotaGa qo-y-i'gowe 'wotaGa che? Ø-na-'begem bo-'ñi? o PIMP-3I-quemar o nomás 3I-sentarse-ARRIB BCS-DSE o lo quemaron, o será que se encendió solo

bo'qa?li

hace tiempo

hace tiempo'

A su vez, cuando el hablante explicita una alternativa al referente o situación respecto de la cual no está seguro se utiliza el coordinante disyuntivo 'wotasa 'o' solo (28)-(29) o junto a bo-DEM (28), (30)-(31). 
Carpio y González • Evidencialidad y modalidad epistémica en dos variedades de toba 143

(29) $n-e^{\prime} k i \mathrm{i} i$

la'qaya ga? wo? da? beyaGa'wa 'wotaGa

3POS.ALIE-alma/foto otro DNP EXIST DPA persona o

'Otra foto en la que había una persona o

noGoto'le-k

niña-MASC

un niño’

(30) $n-e^{\prime} k i ? i$

la'qaya, wo? 'di?-me

biya $a^{\prime} w a$

3POS.ALIE-alma/foto otro EXIST DACOS-ENDOF persona

'Otra foto en la que había una persona

d-oPo'che-tak, wota'Ga i-le'wak bo-'di?

3I-dormir-PROG o 3I-estar enfermo BCS-DACOS

que estaba durmiendo o estaba enferma, ¿será?’

(31) no se si $y$-iwa' Gano-we ha-'da?-me

no se si 3I-golpear-EM FEM-DPA-ENDOF

'No sé si golpea la

ñ-i'bom 'l-apo bo-'ñi? 'wotaga ben'tana'lapo

1POS.INAL-puerta 3POS.INAL-tapa BCS-DSE o ventana 3POS.INAL-tapa

hoja de la puerta, ¿será?, o la hoja de la ventana'

\section{Conclusiones}

Si bien la evidencialidad y la modalidad epistémica habían sido analizadas en estudios previos sobre otras lenguas de la familia guaycurú, las estrategias que hemos presentado en este trabajo no habían sido descritas. Entendemos que en las variedades de toba habladas en Formosa es factible plantear la evidencialidad y la modalidad epistémica como dos categorías distintas, de acuerdo con las estrategias morfosintácticas que permiten codificarlas. Es decir, los datos analizados reflejan, como sostiene De Haan (2001: 201-203), que el vínculo entre la fuente de información y el grado de certeza 
subjetiva constituye una relación de segundo grado, solamente inferida y no necesaria. En toba del este, describimos el recurso a una estructura perifrástica que implica una ruta de gramaticalización del verbo 'decir', como estrategia de evidencialidad indirecta reportativa o citativa, y a adverbios modales con el fin de codificar modalidad epistémica. En toba del oeste, analizamos el uso del prefijo bo- junto a las raíces demostrativas pospuestas al elemento - nombre, verbo, adverbio o cláusula - respecto del cual se expresa incertidumbre. Esta estrategia de codificación de modalidad epistémica contrasta con las bases demostrativas exofóricas visibles y no-visibles, que expresan fuente de información, antepuestas a los nombres modificados. Si bien permiten identificar la fuente de información, estas bases demostrativas no son descritas dentro de la evidencialidad debido a que su alcance se restringe a los referentes de los nominales con los que ocurren o a los que reemplazan como pronombres.

A su vez, hemos demostrado que la construcción que permite codificar evidencialidad de tipo reportativo, en toba del este de Formosa, se encuentra en un proceso de gramaticalización a partir del verbo qoyitega 'dicen', y que tanto este tipo de evidencialidad como las construcciones que permiten introducir citas directas implican el uso de verbos de 'decir'.

La evidencialidad y la modalidad epistémica constituyen dos categorías que deben ser analizadas, no solamente en sus aspectos morfosintácticos y semánticos, sino, además, a nivel pragmático como estrategia discursiva en la interacción comunicacional. En este sentido, será pertinente continuar el análisis, en futuros trabajos, en un corpus de tipo conversacional con el fin de detectar no solo otras estrategias morfosintácticas, sino también de ampliar la observación de los contextos fundamentalmente pragmáticos de uso de las construcciones ya descritas en este trabajo.

Abreviaturas: 1, 2, 3=primera segunda y tercera persona; $1 \mathrm{G}=$ primera persona "grupo"; $\mathrm{I}=$ índice pronominal tipo $\mathrm{I}$; II=índice pronominal tipo II; $\mathrm{AB}=$ direccional 'abajo'; $\mathrm{ADV}=$ adverbio; AGT=afijo 'agentivo'; AL=aplicativo 'alativo'; ALIE=alienable; 
Carpio y González • Evidencialidad y modalidad epistémica en dos variedades de toba 145

ARRIB $=$ direccional 'hacia arriba'; $B C S=$ baja certeza subjetiva; $\mathrm{CE}=$ cambio de estado; $\mathrm{COL}=$ colectivo; $\mathrm{COORD}=$ coordinante; DAC $=$ demostrativo 'acercándose'; DACOS=demostrativo 'acostado'; DAL=demostrativo 'alejándose'; $\mathrm{DDAC}=$ determinante duplicado 'acercamiento'; DDAL=demostrativo duplicado 'alejamiento'; $\mathrm{DEB}=$ aplicativo locativo 'debajo'; DIM=diminutivo; $\mathrm{DIR}_{1}=$ aplicativo direccional 'meta'; $\mathrm{DNP}=$ demostrativo 'no presente'; DPA=demostrativo 'parado'; DSE=demostrativo 'sentado'; $\mathrm{EM}=$ direccional tipo II 'en medio de, entre'; ENDOF=endofórico; EXIST=existencial; EXOFVIS=exofórico visible; $\mathrm{EXT}=$ direccional 'hacia afuera'; FEM=femenino; GENT=gentilicio; ID=identificador demostrativo; INAL=inalienable; IPOS=poseedor indefinido; IT=aspecto iterativo; $\mathrm{LOC}_{2}=$ aplicativo locativo 'adentro'; $\mathrm{MASC}=$ masculino; $\mathrm{ME}=$ modalidad epistémica; $\mathrm{NEG}=$ negación; $\mathrm{NPROG}=$ no progresivo; $\mathrm{PDAC}=$ pronombre duplicado 'acercamiento'; PIMP=pasiva impersonal; PL=plural; PLPA=plural argumento $\mathrm{P}$ aplicado; $\mathrm{POS}=$ poseedor; PROG=progresivo; REL1=relativizador $1 ; \mathrm{SG}=$ singular.

\section{Referencias bibliográficas}

Aikhenvald, Alexandra 2004 Evidentiality. Oxford: Oxford University Press.

Bybee, Joan, Revere Perkins y William Pagliuca

1994 The evolution of grammar. Tense, aspect, and modality in the languages of the word. Chicago y Londres: The University of Chicago Press.

CARPio, María Belén

2012 Fonología y morfosintaxis de la lengua hablada por grupos tobas en el oeste de Formosa (Argentina). LINCOM Studies in Native American Linguistics 67. München: LINCOM Europa Academic Publisher.

Censabella, Marisa

2011 "Gramaticalización del aplicativo posicional -Pot en toba". En Investigaciones sobre lenguas indígenas sudamericanas. Eds., Ana Fernández Garay y Antonio Díaz-Fernández. Santa Rosa: Universidad Nacional de La Pampa, 41-68. 
Chafe, Wallace

1986 "Evidentiality in English Conversation and Academic Writing”. En Evidentiality: The Linguistic Coding of Epistemology. Eds., Wallace Chafe y Joanna Nichols. Norwood: Ablex, 261-272.

Company Company, Concepción

2009 "Parámetros de gramaticalización en los indefinidos compuestos en el español”. En Romanística sin complejos. Homenaje a Carmen Pensado. Ed., Fernando Sánchez Miret. Berna: Peter Lang, 71-104.

De HAAn, Ferdinand

2001 "The Relation between Modality and Evidentiality". Linguistische Berichte. 9, 201-216.

2005 "Encoding speaker perspective: evidentials". En Linguistic Diversity and Language Theories. Eds., Sigmungt Frajzyngier, Adam Hodges y David Rood. Amsterdam/Philadelphia: John Benjamins Publishing Company, 379-397. https://doi. org/10.2307/413372

FABRE, Alain

2006 "Los pueblos del Gran Chaco y sus lenguas, tercera parte: Los guaykurú”. Suplemento Antropológico. 41, 2, 7-132.

Givón, Talmy

2001 Syntax. A functional-typological introduction. Vol. I. Amsterdam/Philadelphia: John Benjamins Publishing Company.

GonZÁlez, Raúl Eduardo

2015 "Estudio fonológico, morfológico y sintáctico del toba hablado en el este de la Provincia de Formosa (Argentina) desde el enfoque tipológico funcional”. Tesis de doctorado en Letras. Universidad Nacional del Nordeste.

Gordillo, Gastón

2006 En el Gran Chaco: Antropologias e bistorias. Buenos Aires: Prometeo Libros.

Heine, Bernd y Tania Kuteva

2002 World Lexicon of Grammaticalization. Cambridge: Cambridge University Press. https://doi.org/10.1017/cbo9780511613463 
Carpio y González • Evidencialidad y modalidad epistémica en dos variedades de toba 147

Instituto Nacional de Estadística y Censos (INDEC)

2012 Censo nacional de población, hogares y viviendas 2010: Censo del Bicentenario. Resultados definitivos. Serie B № 2, Tomo 1. Buenos Aires: Instituto Nacional de Estadística y Censos (INDEC). Consultado: 7 de abril de 2013. <http://www. indec.gob.ar>.

Mendoza, Marcela y Pablo Wright

1989 "Sociocultural and economic elements of the adaptation systems of the Argentine Toba: the Nacilamolek and Taksek cases of Formosa Province". En Archaeological approaches to cultural identity. Ed., Stephen Shennan. Londres: Unwin Hyman, 242-257.

Messineo, Cristina

2003 Lengua toba (guaycurú). Aspectos gramaticales y discursivos. LINCOM Studies in Native American Linguistics 48. München: LINCOM Europa Academic Publisher.

Mushin, Ilana

2001 Evidentiality and epistemological stance: narrative retelling. Amsterdam/Philadelphia: John Benjamins Publishing Company. https://doi.org/10.1075/pbns.87

Siewierska, Anna

1984 The Passive: A comparative Linguistic Analysis. Londres: Routledge.

Tovar, Antonio y Consuelo Larrucea de Tovar

1984 Catálogo de las lenguas de América del sur. Madrid: Gredos.

VIDAL, Alejandra y Harriet E. KLEIN

1998 "Irrealis in Pilagá and Toba? Syntactic versus pragmatic coding”. Anthropological Linguistics. 40, 2, 175-197.

Wright, Pablo

2002 "Ser católico y ser evangelio": tiempo, historia y existencia en la religión toba. Revista Anthropológicas. 6, 13 (2), 61-81.

2008 Ser-en-el-sueño. Crónicas de historia y vida toba. Buenos Aires: Biblos.

Fecha de recepción: 23/08/2015

Fecha de aceptación: 12/04/2016 